DIVISION OF THE HUMANITIES AND SOCIAL SCIENCES

CALIFORNIA INSTITUTE OF TECHNOLOGY

PASADENA, CALIFORNIA 91125

ON ELICITING BELIEFS IN STRATEGIC GAMES

Thomas R. Palfrey

California Institute of Technology

Stephanie W. Wang

Princeton University

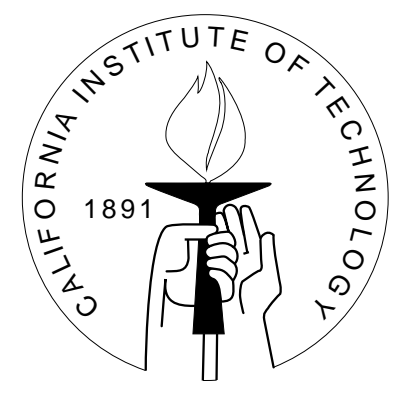

SOCIAL SCIENCE WORKING PAPER 1271R

Revised December 2007 


\title{
On Eliciting Beliefs in Strategic Games ${ }^{1}$
}

\author{
Thomas R. Palfrey ${ }^{2}$ and Stephanie W. Wang ${ }^{3}$
}

March 2007. (Current Version December 14, 2007)

\footnotetext{
${ }^{1}$ We gratefully acknowledge the financial support of the National Science Foundation (SES0079301, SES-0450712, SES-0094800), The Princeton Laboratory for Experimental Social Science, and the Social Science Experimental Laboratory at Caltech. We are grateful for comments from Juan Carrillo, audience members at the 2007 meeting of the Public Choice Society in Amsterdam, and participants at the 2007 conference at the University of Exeter on Risk, Forecast, and Decision.

${ }^{2}$ Division of the Humanities and Social Sciences, California Institute of Technology, Pasadena, CA 91125.

${ }^{3}$ Department of Economics, Princeton University, Princeton, NJ 08540.
} 


\begin{abstract}
Several recent studies in experimental economics have tried to measure beliefs of subjects engaged in strategic games with other subjects. Using data from one such study (Nyarko-Schotter, 2002) we conduct an experiment where our experienced subjects observe early rounds of strategy choices from that study and are given monetary incentives to report forecasts of choices in later rounds. We elicit beliefs using three different scoring rules: linear, logarithmic, and quadratic. There are differences between the elicited beliefs under quadratic and logarithmic scoring rules in spite of both being proper scoring rules. The (improper) linear scoring rule frequently elicits boundary forecasts as theory predicts, and is poorly calibrated. We compare the forecasts of our trained observers to forecasts of the actual players in the Nyarko-Schotter experiment and identify several differences. There was a significant positive correlation between observer forecasts and the choice behavior in the game under both proper scoring rules, but no significant correlation between the players' own forecasts and the actual play. This raises doubts about whether beliefs can be reliably elicited from players who simultaneously have a stake in the target of their forecast, in this case the opponent's choice. The distribution of player forecasts also tended to be more extreme than the observer forecasts using either of the proper scoring rules. We also find evidence of belief convergence when beliefs are elicited iteratively from a group.

Keywords: Scoring rules; Experiment; Game theory; Forecasting; Beliefs
\end{abstract}




\section{Introduction}

Probabilistic beliefs play a central role in mathematical theories of strategic decision making. In games of strategy, optimal decisions depend on beliefs about other players' choices, which in turn depend on their beliefs about one's own decision, and so on. Many ideas lying at the very foundation of these theories and related concepts in economics, such as rational expectations and Nash equilibrium are built around strong assumptions about beliefs. Most attempts to test these theories, often in laboratory experiments, either measure beliefs indirectly by estimation, or impose maintained hypotheses about beliefs (such as rational expectations), resulting in tests of joint hypotheses about beliefs and rational choice. The ability to evaluate or test these theories more sharply would be greatly enhanced if it were possible to measure beliefs directly. Indeed, there has a number of recent attempts of direct measurement of probabilistic beliefs by experimental economists, in the context of strategic games. Examples include Dominitz and Hung (2004), in the context of information cascades, Huck and Weizsacker (2002) in the context of lottery choice experiments, McKelvey and Page (1990) for information aggregation, Duwfenberg and Gneezy (2000) in trust games, Offerman et al. (1996) and Croson (2000) in voluntary contribution games. The results of those papers raise questions about the measurement methodology itself, and its applicability to the

elicitation of beliefs in a strategic environment. Indeed, a striking finding from several of these experiments is the surprising prevalence of extreme forecasts (degenerate or nearly degenerate forecasts), which is hard to reconcile with standard theory.

This paper explores four methodological questions and two substantive questions about the use of scoring rules for the elicitation of probabilistic beliefs about behavior in strategic games. We undertake this exploration in the context of a simple $2 \mathrm{x} 2$ asymmetric matching pennies game similar to the one originally studied by Ochs (1995) and more recently by McKelvey, Palfrey, and Weber (2000), Goeree, Holt, and Palfrey 
(2003), and Nyarko and Schotter (NS, 2002).

The first question is: can beliefs be reliably elicited from the players of a game, during the play of the game? Unreliable reported beliefs could arise for a variety of reasons, including psychological factors such as rationalization, or via distortion of incentives because they are also being paid according their play in the game, which violates the "no-stakes" condition of Kadane and Winkler (1988). We address this question by comparing the elicited beliefs of (experienced) observers to the elicited beliefs of the players themselves. Our subjects observe real sequences of choice behavior from the NS data, and are asked to make probabilistic one-move-ahead forecasts of the play of the game, as the sequence is played back to them in real time, using scoring rules to incentivize the forecasts. Because the NS subjects also made incentivized one-move-ahead forecasts, this allows for a direct comparison.

The other three methodological questions address the issue of whether the choice of the scoring rule makes a difference: Are forecasts elicited using proper scoring rules systematically different from those elicited using improper scoring rules? Are forecasts elicited via two different proper scoring rules the same or different? Are forecasts better calibrated for some scoring rules than others? With these latter two questions in mind, we conduct an experiment with three different treatments, each corresponding to a different scoring rule. The three scoring rules used are logarithmic (proper), quadratic (proper), and linear (improper).

The substantive questions both concern information aggregation and belief convergence of subjective beliefs. First, are individuals in a group able update their beliefs in response to the forecasts of other members of the group? (belief convergence) Second, if such convergence occurs, are individual forecasts improved by group interaction? (information aggregation) To address these questions, our experiment includes a second feature that allows for information aggregation. Our observers were 
placed in groups of four, and there were two sequential rounds for each forecast. The entire profile of individual forecasts of group members was revealed between the two rounds, so each individual had an opportunity to update his or her forecast in response to the forecasts of the other group members. This allows us to test for belief convergence (comparing the variance of first round to second round forecasts) and information aggregation (comparing the accuracy of first round and second round forecasts).

We have five main findings. First, there is a difference between the elicited beliefs under quadratic and logarithmic scoring rules in spite of both being proper scoring rules. Forecasts elicited from our observers using the logarithmic scoring rule are less extreme (closer to $(.5,5))$ than forecasts under the quadratic scoring rule according to several different measures. Second, the linear scoring rule produces forecasts closer to 0 and 1 than the proper rules, and these forecasts are poorly calibrated. Third, the forecasts by our observers with both proper scoring rules were more accurate than the forecasts of the NS players, in the sense that the average elicited forecast was closer to the true choice frequencies in the data. Furthermore, there was a significant positive correlation between observer forecasts and the choice behavior in the game under proper scoring rules, while there was no significant correlation between the NS players' forecasts and the actual play. This reinforces doubts about whether beliefs can be reliably elicited from players who simultaneously have a stake in the target of their forecast, in this case his opponent's choice. Fourth, the distribution of forecasts by NS players were more extreme than the observer forecasts using either of the proper scoring rules. Fifth, we find evidence for belief convergence among our observers. 


\section{$1.1 \quad$ Related Literature}

\subsubsection{Scoring rules}

Scoring rules, which yield payoffs as a function of vector of probabilistic forecasts and a realized event, are used to elicit subjective probabilities in laboratory and real-life settings. Different scoring rules have different incentive compatibility properties. Because elicitation methods are used to uncover "true" probabilistic beliefs, incentive compatibility is an important criterion for the "goodness" of any scoring rule. A scoring rule is classified as proper if it is incentive compatible. In the scoring rule literature, a scoring rule is considered incentive compatible if a forecaster cannot attain a higher expected score by reporting a probability different than her true probability.

Brier (1950) and Good (1952) were the first to identify two such proper scoring rules, quadratic and logarithmic, respectively. Since then, both the quadratic and logarithmic scoring rules as well as others have been shown to be strictly proper. Savage (1971) specifies the general rule for generating the class of strictly proper scoring rules and there have been numerous theoretical studies of desirable and undesirable properties of proper and improper scoring rules.

\subsubsection{Previous experiments using scoring rules to elicit beliefs}

The quadratic scoring rule is the most common one applied in both laboratory and field experimental settings for the forecasting of subjective events such as weather forecasting (Staël von Holstein 1971), stock market prices (Staël von Holstein 1972), outcomes of sporting competitions (Winkler 1971), and game theory (see below). The logarithmic scoring rule has been applied to a much lesser extent in experiments on education testing (Hambleton et. al. 1970; Glein and Wallace 1974) and information aggregation (Ledyard et. al. 2005).

A few articles in the psychology literature have studied belief elicitation with 
different scoring rules, but none has conducted a comprehensive comparison of elicitations from the logarithmic, quadratic, and linear scoring rules, none have looked at the use of scoring rules for belief elicitation in the context of strategic choices in games, and none have compared player and observer forecasts.

Studies in experimental economics that have tried to use of scoring rules to elicit subjective beliefs about action choices in a strategic game have produced mixed results. In the context of two-person matrix games, extreme reported beliefs are observed with surprising frequency (Dominitz and Hung 2004, Nyarko and Schotter 2002). Because the "true" frequencies of target states is generally between .35 and .65 in these studies, this suggests bias in the forecasts. Furthermore, beliefs are erratic, in the sense that they change much faster from period to period than a Bayesian model would predict, indicating that forecasts are not only inaccurate, but highly imprecise (Nyarko and Schotter 2002, fig. 2, p. 980). If the players were adjusting beliefs according to Bayes rule or even according to a simple counting procedure, truthful reporting of beliefs should have a smoother trajectory than the observed forecasts. There is also evidence from two person laboratory games that the process by which subjects decide on a forecast is qualitatively different from the decision process they use to make a decision, which can sometimes result in forecasts that are inconsistent with choice behavior (Costa-Gomes and Weizsacker 2006).

In contrast, Dominitz and Hung (2004), in the context of an information cascade experiment, report that players' forecasts are dampened relative to Bayesian reports. In particular, they find that subjects often fail to change their forecasts in response to hard information, which suggests possible distortions in the elicitation procedure. The task was different from the our task of one-step-ahead forecasts of choices in a repeated game, since their subjects were repeatedly forecasting a static target (the state of the world), rather than a stochastically moving target. Offerman et al. (1996) elicited subjective 
player forecasts about the level of contributions of other players in a voluntary contributions game. Some of the forecasts were degenerate, bimodal, or implausible for other reasons, and they confirm the finding reported by Palfrey and Rosenthal (1991) that subject beliefs about others' contributions exhibit an optimism bias.

There is very little evidence about the similarities and differences between forecasts elicited from observers and forecasts elicited from players themselves, and what evidence exists is mixed. Huck and Weizsacker (2002) elicit forecasts from subjects who observe decision makers in a simple (objective) binary lottery choice task. They find some inaccuracies, notably that the forecasts are closer to 50/50 than the actual choice frequencies of the subjects, and that this doesn't depend in a significant way on the elicitation procedure. This is in stark contrast to the forecasting behavior measured using an identical quadratic scoring rule in the NS experiment, where reported beliefs of players are biased in the opposite direction. These two findings are also at odds with findings reported in Offerman et al. (1996, p. 828), where observers submitted forecasts that were more extreme than those submitted by the players themselves.

\subsubsection{Convergence of beliefs}

Our iterative elicitation method could induce a common knowledge inference process whereby individual beliefs adjust after others' beliefs are revealed. In the common knowledge literature, Aumann (1976) first established that if two agents have the same common prior, their posterior probability of an event must be the same if the posteriors are common knowledge. The subsequent work of Geanakoplos and Polemarchakis (1982), McKelvey and Page (1986), and Nielsen et al. (1990) are more closely related to the possible process generated by our iterative elicitation method. Geanakoplos and Polemarchakis show that with iterated exchange of information between the agents, the inference process would terminate at a point where the posterior probabilities are equal. 
Related to our iterative elicitation method are experiments in which subjects receive feedback about other subjects' forecasts (McKelvey and Page 1990; Offerman and Sonnemans 1998; Winkler 1968). With the exception of Winkler's experiment in which he elicits forecasts about subjects with intrinsic uncertainty such as the weather or sports through an unincentivized questionnaire, the rest induced differences in private information in the laboratory and focused upon the efficiency of private information pooling when there is objective uncertainty. These studies report some belief convergence as measured by the reported forecasts of these objective events.

\section{Theoretical Background}

\subsection{Simple Matrix Game}

This is the simple matrix game that was used in the Nyarko-Schotter experiment and in ours as well.

\begin{tabular}{|l||l|l|}
\hline & Green & Red \\
\hline \hline Green & 6,2 & 3,5 \\
\hline Red & 3,5 & 5,3 \\
\hline
\end{tabular}

Table 1. Matrix game payoffs.

This is a constant sum game with an unique Nash equilibrium in mixed strategies that is supported by the principle of best response. In equilibrium both players choose Green with $40 \%$ probability and Red with $60 \%$ probability.

\subsection{Three Scoring Rules}

Scoring rules, which compute a numerical score as a function of the stated probabilities as well as the realized event, are often used in forecasting and experimental settings to assess the accuracy of forecasts. In our experiment, this score also specifies the monetary payoff. A scoring rule is proper if the forecaster maximizes her expected monetary 
payoff by revealing her true belief. We next describe the three scoring rules used in the three belief elicitation treatments of our experiment. We then go on to show that the quadratic and logarithmic scoring rules are proper whilst the linear scoring rule is not.

\subsubsection{Preliminaries}

Let $i=1,2, \ldots, n$ denote the $n$ possible events and let $p=\left(p_{1}, p_{2}, \ldots, p_{n}\right)$ be the forecaster's stated forecast, where $p_{i}$ is the stated probability of event $i$. Define the scoring rule $S=\left\{S_{1}, S_{2}, \ldots, S_{n}\right\}$ as a collection of scoring functions where $S_{i}(p)$ specifies the score when event $i$ is realized as a function of the forecast, $p$. Let $\pi=\left(\pi_{1}, \pi_{2}, \ldots, \pi_{n}\right)$ be the subject's true belief where $\pi_{i}$ is the probability of event $i$.

\subsubsection{Characterization}

1. Quadratic Scoring Rule:

$$
S_{i}(p)=\alpha-\beta \sum_{k=1}^{n}\left(I_{k}-p_{k}\right)^{2}
$$

where $\alpha, \beta>0$ and $I_{k}$ is an indicator function that takes the value 1 if the realized event is event $k$ and 0 otherwise. The quadratic rule scores the inaccuracy of the forecast as a constant minus the sum of the square deviations. In our belief elicitation experiment, there two possible events the observer is forecasting: the event that the player being observed chooses Green, which we denote as $G$, or Red, $R$. We denote the two forecasts by $p_{G}$ and $p_{R}$, respectively, where $p_{G}+p_{R}=1$. Following Nyarko-Schotter (2002), we pay our subjects in the quadratic treatment an amount in dollars that is proportional to their score, using parameters $\alpha=1$ and $\beta=0.5$. The score is therefore:

$$
\begin{aligned}
& S_{G}=1-p_{R}^{2} \text { if } G \text { is chosen } \\
& S_{R}=1-p_{G}^{2} \text { if } R \text { is chosen }
\end{aligned}
$$


It is a straightforward exercise to prove that the quadratic rule is proper: i.e., a forecaster with true beliefs $\pi$ maximizes expected score (expected payoff) by reporting $p=\pi$.

\section{Logarithmic Scoring Rule:}

$$
S_{i}(p)=\alpha+\beta\left(\log p_{i}\right)
$$

where $\alpha, \beta>0$

The logarithmic rule, which is also proper, equals a constant less a penalty proportional to the natural log of the forecast of the realized event (a negative

number since $0 \leq p_{i} \leq 1$ ). The lower the forecast of the realized event, the greater is the penalty. The score is $-\infty$, however, when zero probability is placed on the realized event. Because of this property, implementation in practice requires bounding the forecasts away from zero. We place bounds on the maximum (0.9) and minimum (0.1) probability a subject can place on any event. We set $\alpha=1$ and $\beta=0.45$. The score if event $i$ occurs in the logarithmic treatment is:

$$
S_{i}(p)=1+0.45\left(\log p_{i}\right)
$$

3. Linear Scoring Rule:

$$
S_{i}(p)=\alpha+\beta p_{i}
$$

where $\beta>0$

We use $\alpha=0$ and $\beta=1$ in our experiment, so the linear score is simply the probability forecast for the realized event. The linear scoring rule is not proper. A forecaster with true beliefs $\pi$ maximizes expected linear score by placing maximum weight on the most likely event. If the forecaster believes the two events are equally likely, then any forecast is optimal. 


\section{Experimental design and procedures}

We conducted six sessions with a total of 48 subjects. Subjects were registered students at Princeton University, and were recruited by E-mail solicitation. Sessions were conducted at the Princeton Laboratory for Experimental Social Science, and all interaction was computerized. Each subject participated in exactly one session, with 8 subjects per session. The primary treatment variable was the scoring rule, either log, quadratic, or linear, with one third of the subjects in each treatment.

Each session had two parts. Instructions were read aloud to the subjects. ${ }^{1}$ In the first part, subjects were randomly assigned to be either the row player or the column player in the 2x2 game in Table 1. Keeping the pairings fixed, they played the game repeatedly for 5 rounds. After round 5 , they are assigned to the opposite role so that if they were a row player in the first five rounds, they are now a column player and vice versa. They are also randomly repaired with a different player and play the game repeatedly for 5 rounds with this new opponent. Their earnings for Part 1 was the sum of their earnings over all 10 rounds of play. The sole purpose of part 1 of the session was to give subjects experience with the game.

In part 2, subjects did not play the game, but instead made "observer" forecasts about the sequence of choices of either the row or the column player in seven different pairs from the Nyarko-Schotter (NS) experiment. In each session, four subjects (row forecasters) were assigned the task of sequentially forecasting choices of NS row players and the other four subjects (column forecasters) were assigned the task of forecasting the choices of NS column players. These roles were fixed throughout part 2. The scoring rule (quadratic, log, or linear) was fixed throughout the session, and was explained carefully to the subjects.

We then played back the data sequentially to the subjects in the following way.

\footnotetext{
${ }^{1}$ A sample copy of the instructions is available http://www.hss.caltech.edu/ ${ }^{\sim}$ trp/elicit/sample_instructions.pdf.
} 
First, for one particular NS pair, all eight observers are told the actions chosen by the two players of a particular NS pair in the first five rounds of that match. The list of actions chosen by that NS pair in the first five matches is displayed on every subject's computer screen. Each row forecaster is then asked to report a forecast about the likelihood the row player in that pair chose red or green in round six, and column forecasters are asked to report a forecast about the likelihood the column player in that pair chose red or green in round six. This is implemented by requiring each subject to type in two integers, one for green and one for red, where the two numbers must add up to $100 .^{2}$ All the column predictors simultaneously and independently make forecasts in this manner about the actions of the one column player in round 6 of that NS pair, and all the row forecasters simultaneously and independently make forecasts in this manner about the actions of the one row player of the same NS pair.

After reporting these forecasts, all row forecasters are told the forecasts of all the other row forecasters, and all column forecasters are told the forecasts of all the other column forecasters. We then elicit a second forecast from each subject by the same method. This second forecast can be the same or different from the first forecast.

After the revised forecasts have been made, the actual choices by the row and column players in round 6 of that NS pair are then reported back to the subjects, so they now know the choices by both subjects in the first six rounds of the match. For each subject, one of their two forecasts was randomly chosen for actual dollar payoff.

Subjects then proceed to make forecasts about round 7 of that NS pair, in the same manner as they made forecasts about round 6. Roles (row or column forecaster) stay fixed. They continue in this way to make iterative forecasts for the play in rounds 8,9 , and 10 of that NS pair, receiving feedback after each forecast. This procedure was then

\footnotetext{
${ }^{2}$ Because the log scoring rule gives negative payoffs at the boundary ( 0 or 100$)$, forecasts for that scoring rule were constrained to be betweeen 10 and 90 . For consistency, the same constraint was imposed with the other scoring rules.
} 
repeated (sequentially) during the session so that the eight subjects observed a total of 7 NS pairs. Thus, overall, subjects reported and revised forecasts sequentially for a total of 35 plays of the game by 7 different pairs. They were paid the sum of their dollar payoffs in all 35 rounds. Total earnings ranged from $\$ 17$ to $\$ 35$.

\section{Results}

We analyze the results in two subsections. First, we describe the main aggregate features of the initial elicitation data, before subjects have had the chance to revise their forecasts in light of the forecasts of others. We compare the distribution of forecasts across treatments and across roles. We also compare our data with the distribution of forecasts elicited from NS subjects in rounds 6-10 of that experiment and to the aggregate frequency of choices observed in their data.

Second, we analyze the accuracy of the forecasts. We use two benchmarks: uninformed forecasting (always forecasting 50/50) and rational expectations (forecasting the empirical average frequency in every round). We refer to 50/50 forecasts as uninformed because such a report is optimal for a forecaster whose prior is uniform on $[0,1]$.

Third, we investigate questions about the iterative elicitation process. Does it lead to convergence of beliefs? Does the iterative process lead to more accurate forecasts?

\subsection{Individual forecasts: Comparison of scoring rules and comparison with NS}

Table 2 compares the average forecasts and the actual choice frequencies, broken down by scoring rule and by role (row or column). ${ }^{3}$ In this and subsequent tables, "column" refers to column moves or forecasts about column moves. "Row" refers to row moves or

\footnotetext{
${ }^{3}$ The analysis in this section considers only the first elicited forecast of subjects. These beliefs are made before they know the forecasts of the other members of their group. We analyze the revised forecasts in the next section, where we address questions of convergence of beliefs and information aggregation.
} 
forecasts about row moves. The first three columns give the average forecast under our three scoring rule treatments. The fourth column is the average forecast in rounds 6-10 of NS experiment 1 (i.e. the same rounds our subjects were forecasting), and the final column gives the actual choice frequencies in those rounds.

Three results are illustrated by this table. First, the NS players and our own subjects systematically underestimate the probability column will choose green and overestimate the probability that row will choose green, but these differences are not significant. Second, this bias is less in both observer treatments with a proper scoring rule, and for both player roles, compared to the NS elicitation from the actual players. ${ }^{4}$ Third, for observers, the bias is less with the proper scoring rules than with the linear scoring rule.

$\begin{array}{rrrrrr} & \text { Quad } & \text { Log } & \text { Lin } & \text { NS Quad } & \text { Observed } \\ \text { Column } & 45.7^{*} & 47.7^{*} & 39.8 & 44.3 & 55.7 \\ \text { Row } & 48.8^{*} & 47.4^{*} & 51.7^{*} & 53.0 & 42.9 \\ \text { N } & 560 & 560 & 560 & 140 & 140\end{array}$

Table 2. Average forecasts compared to observed choices. Entries are \% Green. $*=$ less biased than NS forecasts.

Another way to compare the forecasts of our observer subjects with the forecasts of the actual players of the game is to look at raw correlations between the two. The first row of Table 3 reports these raw correlations using the average first round forecasts of each of our groups of four subjects, matched with the forecasts of the corresponding NS subject. We find large positive correlations for our quadratic scoring rule treatment, less

\footnotetext{
${ }^{4}$ Because the observer forecasts were limited to the range of 10 and 90 and the NS forecasts were not, we were concerned that theses and other results comparing observer forecasts with NS forecasts might be an artifact of this truncation. As a check, we have replicated the analysis of NS forecasts in Table 2 by recoding forecasts that are more extreme than 10 and 90, as 10 and 90, respectively. The results are the same. A similar replication was done for Tables 4 and 5 as well.
} 
so for the linear rule, and actually negative for the log rule. To test for significance of these differences, we ran a Tobit regression of the mean of our elicited beliefs under quadratic treatment on the corresponding NS elicitations. The coefficients and standard errors are reported in the second row of Table 3. The coefficient is significant at the $5 \%$ level only for the quadratic treatment. We cannot reject the hypothesis that our log and linear elicitations are uncorrelated with the elicited beliefs of the NS players.

$\begin{array}{rrrr} & \text { Quad } & \text { Log } & \text { Linear } \\ \text { Correlation } & 0.17 & -0.0081 & 0.072 \\ \text { Tobit Coefficient } & 0.087^{*}(0.043) & -0.0022(0.023) & 0.037(0.043)\end{array}$

Table 3. Correlation between average Observer forecasts and matched NS forecasts. Standard errors in parenthesis. ${ }^{*}=$ significant at $\mathrm{p}=.05$.

The results from Tables 2 and 3 show that the three scoring rules we use with observers clearly do lead to different measurements of beliefs. ${ }^{5}$ To explore this further, we examine the differences in extremeness of elicited beliefs across our three measures and look at how these dispersions compare with the NS elicitations. To measure extremeness, we compute the absolute differences from 50 for each individual forecast. According to the theoretical results, we know that quadratic and log are both proper scoring rules, so we hypothesize no significant difference between the dispersion in forecasts for log and quadratic. In contrast, the linear scoring rule is not proper; indeed, optimizing risk neutral subjects will report beliefs equal to either 0 or 1 . We hypothesize the linear elicitation procedure will result in greater dispersion than the quad or log methods.

In addition, if the distribution of beliefs are the same for observers and players of the game, and if there are no distortions created by having a subject report forecasts

\footnotetext{
${ }^{5}$ As is standard, we maintain a "belief independence" hypothesis throughout. That is, the scoring rule may affect forecasts, but do not directly affect beliefs.
} 
and choose actions at the same time (as in NS), then there should be no difference between the player forecasts and the observer forecasts, at least under the quadratic scoring rule. Thus, we hypothesize that there will be no differences in dispersion between NS forecasts and the observer forecasts using quadratic rule.

The average extremeness across all forecasts in each treatment is reported in Table 4, with the complete CDF of the differences displayed in Figure 1.

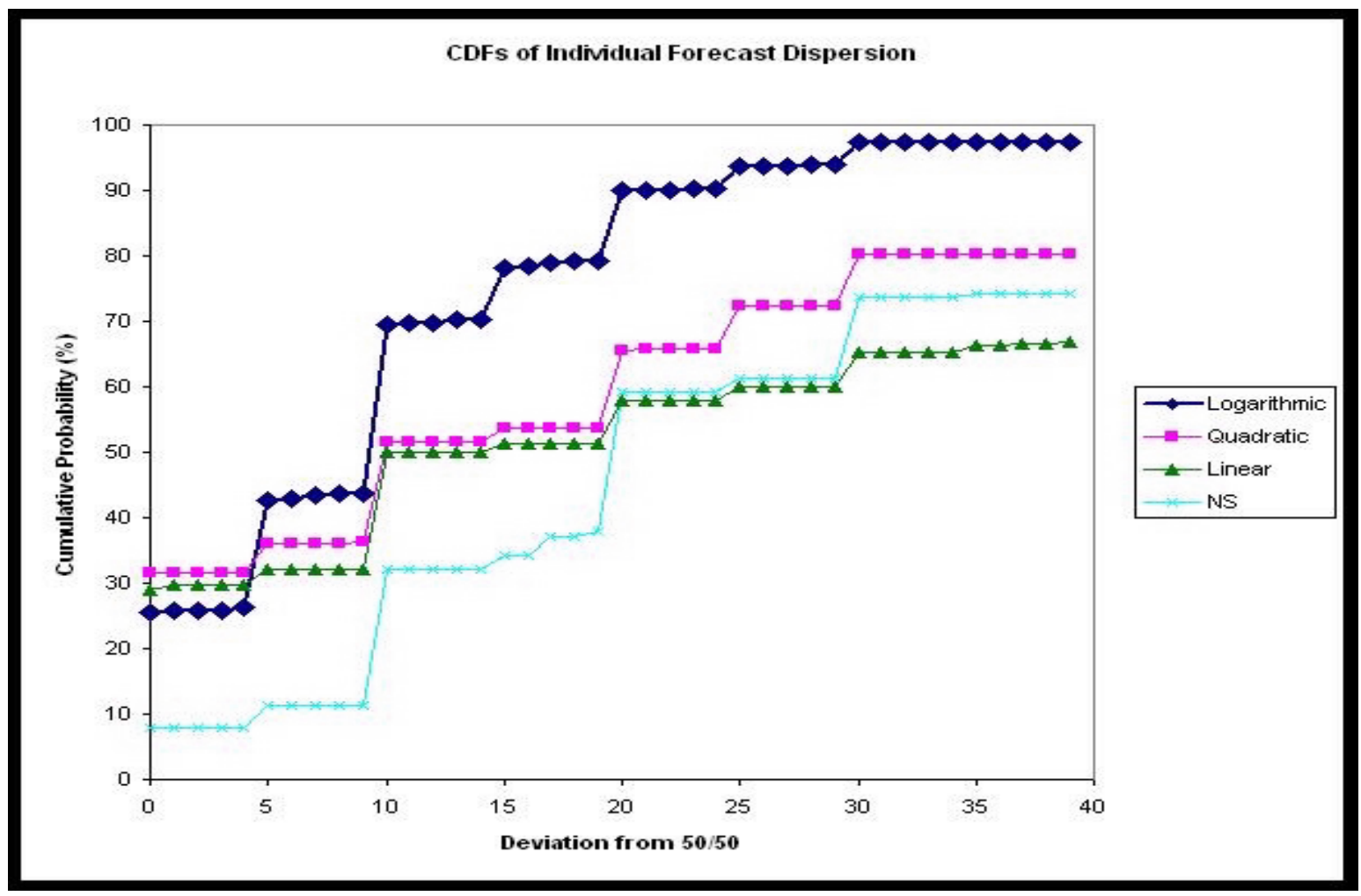

Figure 1. Individual forecast dispersions under the three scoring rules and NS.

The differences are striking. First, the NS player forecasts and linear forecasts exhibit more dispersion than the forecasts by observers with proper scoring rules. The differences are not only significant, but large in magnitude, with the NS dispersions more than double the log scoring rule dispersion and $35 \%$ greater than the dispersion of 
observer quadratic elicited beliefs.

Second, the linear scoring rule leads to the greatest dispersion among the observers, with the comparison to log and quadratic significant as theory predicted. ${ }^{6}$ Third, the linear forecasts are less dispersed than the NS forecasts. Fourth, observer forecasts using quadratic and log scoring rules are significantly different from each other, with the dispersion under the quadratic scoring rule $60 \%$ more than under the log scoring rule.

$\begin{array}{rrrrr} & \text { Quad } & \text { Log } & \text { Linear } & \text { NS } \\ \text { Extremeness } & 16.42^{*}(0.64) & 10.17^{*}(0.40) & 19.38^{*}(0.71) & 22.19(1.12)\end{array}$

Table 4. Forecast extremeness: average absolute difference from 50.

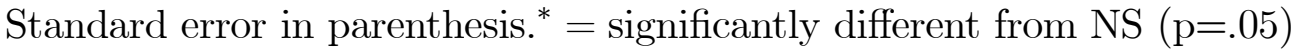

Two other features of the distribution of extremeness are worth noting. The first is stochastic dominance. The distribution of extremeness for the NS player forecasts stochastically dominates the corresponding distributions for both proper scoring rules. Comparing the two proper scoring rules, the distribution of the quadratic rule stochastically dominates the logarithmic rule, except for an insignificant difference at 0 . And both proper scoring rules are stochastically dominated by the improper rule (again with an insignificant difference at 0 ). The only comparison that does not show stochastic dominance is between the observer forecasts with a linear rule and the NS player forecasts. The second feature about the distributions of interest is the frequency of boundary forecasts (i.e., forecasts of $10 \%$ or $90 \%$ ). The NS and linear elicitation procedures resulted in the most boundary forecasts $(25.7 \%$ and $33.2 \%)$, with the proper scoring rules having significantly fewer (19.6\% for quadratic and $2.5 \%$ for logarithmic).

\footnotetext{
${ }^{6}$ This is consistent with Nelson and Bressler (1989) who found that a linear scoring rule generated more extreme forecasts than a quadratic scoring rule.
} 


\subsubsection{Accuracy of reported beliefs: Do the subjects know anything?}

In the actual NS experiment, and also in our experiment using trained observers, the subjects clearly think they know something. Over $92 \%$ of the time, they report "informed" beliefs, i.e., forecasts different from 50/50. In contrast, observers report uninformed beliefs relatively frequently: between $1 / 4$ and $1 / 3$ of the time, depending on the scoring rule.

It is then natural to ask whether the apparently greater confidence of the players is justified. The evidence suggests it is not. We document this in detail below, but the bottom line is apparent from Table 2 in the previous section that shows NS forecasts of row and column actions to be systematically biased and on the wrong side of 50/50. Moreover, because the choice behavior aggregate frequencies hover around $50 \%$ green, extreme forecasts seem to be harder to defend as "rational", compared with fully hedged forecasts.

In contrast, we find evidence that the trained observers with proper scoring rules seem to have some forecasting ability. First we look at the raw correlation between forecasts and the choices they are forecasting. These are given in the first row of Table 5. The overall correlations between forecasts and actions is significantly positive for both observer treatments with proper scoring rules. In contrast, we cannot reject the hypothesis that overall NS forecasts and actions are completely uncorrelated.

$\begin{array}{rrrrr} & \text { Quad } & \text { Log } & \text { Linear } & \text { NS } \\ \text { Correlation } & 0.135^{*} & 0.085^{*} & -0.085^{*} & 0.022 \\ \text { Calibration regression coefficient } & 0.30^{*}(0.09) & 0.31^{*}(0.15) & -0.17^{*}(0.06) & 0.034(0.15) \\ \text { Calibration regression constant } & 34.88^{+}(4.94) & 34.50^{+}(7.64) & 56.96(4.35) & 47.64(8.40)\end{array}$

Table 5. Correlation between individual elicited forecast and actual choice in the experiment.

${ }^{*}$ Coefficient significantly different from $0(\mathrm{p}=.05)$. Standard errors in parenthesis. ${ }^{+}=$constant term significantly different from $50(p=.05)$ 
Second, we ask how well calibrated the forecasters are (Seidenfeld 1985). By Seidenfeld's definition, "a set of probabilistic predictions are calibrated if $p$ percent of all predictions reported at probability $p$ are true." A subject is perfectly calibrated in our experiment if for all the instances when she forecasted Green being played with $30 \%$ probability, Green is played $30 \%$ of the time, for all the time when she forecasted Green being played with $60 \%$ probability, Green is played $60 \%$ of the time, and so on. Table 6 shows the frequency of Green green choice for each forecast (pooled into bins 0-10, 11-20, etc.), with the number of observations in parenthesis. It is clear from Table 6 that the NS forecasts and the ones under the linear scoring rule are badly calibrated.

$\begin{array}{rllrr}\text { Forecast Bin } & \text { Quadratic } & \text { Logarithmic } & \begin{array}{r}\text { Linear } \\ \text { NS }\end{array} & \text { No } \\ 0-10 & 36.1(71) & 30.0(10) & 53.1(113) & 50.0(16) \\ 11-20 & 42.9(28) & 35.7(14) & 48(25) & 41.7(12) \\ 21-30 & 44.9(49) & 46.0(50) & 45.5(22) & 45.5(22) \\ 31-40 & 41.2(51) & 47.2(125) & 57.1(77) & 55.6(18) \\ 41-50 & 52.4(189) & 47.9(192) & 48.3(174) & 46.2(13) \\ 51-60 & 53.6(56) & 56.3(103) & 61.8(34) & 47.1(17) \\ 61-70 & 51.3(39) & 48.9(47) & 38.1(21) & 50(14) \\ 71-80 & 71.1(38) & 86.7(15) & 47.4(19) & 71.4(7) \\ 81-89 & (0) & (0) & 50(2) & 0(1) \\ 90-100 & 51.3(39) & 0(4) & 37.0(73) & 50(20)\end{array}$

Table 6. Calibration: Observed Percent Green Choice by Forecast.

In order to make statements about the statistical significance of calibration, we ran Calibration regressions of the action taken (100 for Green, 0 for Red) on the (first round) forecasts of Green being played. The coefficient on the action choice would be 1 and the intercept 0 if the subjects are perfectly calibrated. The coefficient would be 0 and the intercept 50 if the subjects are forecasts are perfectly uninformed. As reported in the second and third rows of Table 5, we find that the coefficients are significantly greater than 0 and the intercept is significantly less than 50 for the observer treatments with proper scoring rules. In contrast, the coefficients are not significantly greater than 
0, and the constant terms are not significantly less than 50 for either the NS players or the observers using an improper scoring rule.

\subsection{Learning from others' forecasts}

Our experiment had two key design features that allow us to look at questions of information aggregation. First, for each action decision to be forecast, we elicited forecasts from four trained observers, rather than just one. Second, there were two rounds of forecasts, and each forecaster was advised of the forecasts by the other forecasters before reporting a second round forecast. In this section, we address two specific questions about the effects of group feedback on forecasts and how the answers depend on the scoring rule.

1. Do subjects update their forecasts after learning others' forecasts? (belief convergence)

2. Are updated forecasts more accurate? (information aggregation)

\subsubsection{Belief Convergence}

To address question 1, we first compute the frequency that subjects change their forecast in the second round after being told the other forecasters' reports, and the average revision. The findings are reported in Table 7. The answer is yes, forecasters revise their reports in response to the reports of other forecasters. The frequency of revision ranges approximately $1 / 3$ to $1 / 2$, and the average absolute change is significantly positive for all three scoring rules.

$\begin{array}{rrrr} & \text { Quad } & \text { Log } & \text { Linear } \\ \text { Frequency } & 0.37 & 0.57 & 0.32 \\ \text { Average change } & 6.18^{*}(0.53) & 5.72^{*}(0.33) & 8.73^{*}(0.80)\end{array}$

Table 7 . Frequency of and average revisions. ${ }^{*}=$ significantly different from $0(\mathrm{p}=.05)$ 
As further evidence, we look at the change in the variance of forecasts in the group, defined as the variance of second stage forecast minus variance of first stage forecast. If the forecasts are closer together in the second round (negative change in variance), we take that to be evidence of belief convergence. The first three rows of Table 8 display the percent of times the change was negative, zero, or positive, by scoring rule. We find that the within-group variance declines from the first round to the second round about half the time for all three scoring rule treatments, and declines more frequently than it increases. The last row of Table 8 gives the average change in variance for each scoring rule. For all scoring rules, the average change is significantly negative (at $5 \%$ level).

$\begin{array}{rrrr} & \text { Quad } & \text { Log } & \text { Linear } \\ \text { \% Less Variance } & 51 & 49 & 44 \\ \text { \% No Change } & 24 & 4 & 29 \\ \text { \% More Variance } & 24 & 48 & 28 \\ \text { Average change } & -64.30^{*} & -13.69^{*} & -35.57^{*}\end{array}$

Table 8. Directional change in Variance. ${ }^{*}=$ significantly different from $0(\mathrm{p}=.05)$

\subsubsection{Information Aggregation}

To address question 2, we look at the difference between the mean squared deviation (MSD) of initial forecasts and actions versus revised forecasts and action. The first three rows of Table 9 display the percent of times the change (revised minus initial) was positive, zero, or negatively, respectively, by scoring rule. We find that revised forecasts are more accurate by this measure than initial forecasts, but the differences are not large. The last row of Table 9 shows the average change in mean square deviation of forecasts from action (revised minus initial). The changes are negative in all cases, but the magnitudes are small.

\begin{tabular}{crrr} 
\% More Accurate & Quad & Log & Linear \\
\% No Change & 63 & 31 & 18 \\
\% Less Accurate & 18 & 26 & 68 \\
Average change & -0.0040 & -0.0012 & -0.0012 \\
\multicolumn{3}{c}{ Table 9. Directional change in MSD. }
\end{tabular}




\section{Conclusions}

The experiment reported here produced several findings on the elicitation of beliefs with scoring rules. First, the forecasts by our observers under both proper scoring rules were less biased than the forecasts of the NS players, in the sense that the average elicited forecast was closer to the true choice frequencies (Table 2). Second, there was a significant positive correlation between observer forecasts and the choice behavior in the game for both proper scoring rules, while there was no significant correlation between the players' forecasts and the actual play being forecasted; and the correlation was actually negative for the improper scoring rule (Table 5). Third, the distribution of forecasts by NS players were more extreme than the observer forecasts using either of the proper scoring rules. The average NS player forecast deviations (differences from 50/50) were not significantly different from forecasts elicited from observers under the linear scoring rule. Fourth, the linear scoring rule elicits that are significantly more extreme than the two proper rules. Fifth, there is a significant difference between the elicited beliefs under quadratic and logarithmic scoring rules in spite of both being proper scoring rules. Forecasts elicited by the logarithmic scoring rule have significantly less dispersion. Sixth, forecasts elicited under the proper scoring rules were significantly more accurate and better calibrated than those elicited from players and from observers using an improper scoring rule. We did not observe differences in accuracy or calibration for the two proper scoring rules. The relative accuracy of the two varied across our different accuracy measures. Seventh, we find significant evidence for belief convergence but only marginal evidence for information aggregation.

A number of conclusions can be drawn from these findings. We summarize our findings in terms of the answers they give to the four methodological questions and two substantive questions posed in the introduction of the paper. 
1. Can beliefs be reliably elicited from the players of a game, during the play of the game? The first two findings described above raise serious doubts about the reliability of beliefs elicited from players who simultaneously have a stake both in the accuracy of their forecast and in the outcome itself, in this case an opponent's choice in a two person game. The third finding suggests what one might call an overconfidence bias in player-forecasts, relative to the forecasts of experienced observers. A deeper exploration into the sources of this bias is an interesting topic for future research. A possible explanation is rationalization: players report forecasts that reinforce their choice.

2. Are forecasts elicited using proper scoring rules systematically different from those elicited by improper scoring rules? Yes, as implied by the sixth finding listed above. Both proper scoring rules elicit forecasts from our observers that are significantly more accurate and better calibrated than those elicited under the linear scoring rule. One source of the bias caused by linear forecasts is that it elicits more extreme forecasts, as predicted by standard theory.

3. Do different proper scoring rules elicit similar forecasts? Yes. The main difference between forecasts elicited under logarithmic and quadratic scoring rules was that the quadratic rule elicited more extreme beliefs than the logarithmic rule. The distribution of extremeness of forecasts under the quadratic rule stochastically dominates the distribution under the logarithmic rule. It is interesting that this did not result in either one eliciting more accurate or better calibrated forecasts on average than the other. Why we observe this difference is an open question. The procedures used were identical, except for the scoring rule, and it seems implausible that the difference is due to subject heterogeneity and sampling variation. Risk aversion is not a plausible explanation either. While risk aversion 
can distort reported forecasts, if subjects have constant relative risk aversion, there is virtually no difference in the theoretical distortion that would result under the two rules. Loss avoidance may be a possible explanation for the difference in boundary forecasts, but cannot explain the stochastic dominance finding. Other possibilities, such as ambiguity aversion and other violations of expected utility theory are worth pursuing in future research, but are beyond the scope of this paper.

4. Are elicited forecasts more accurate and/or better calibrated under some scoring rules than others? Yes. Forecasts from proper scoring rules are more accurate and better calibrated than forecasts from improper scoring rules.

5. Do individuals in a group update their beliefs in response to the forecasts of other members of the group?

We found significant forecast revisions in all three scoring rule treatments. The within group variance of revised forecasts is significantly less than the variance of initial forecasts. We infer from this that beliefs are converging.

6. Are individual forecasts improved by group interaction? Revised forecasts are more accurate than initial forecasts, as measured by the MSD, but the magnitude of improvement is small and statistically insignificant.

The choice of scoring rule to elicit probabilistic beliefs about subjective events can make a difference. The distribution of our elicited beliefs under the three scoring rules are significantly different from each other. Our findings add to evidence elsewhere that the elicitation of beliefs directly from players, simultaneously playing the game for which they are forecasting outcomes may be unreliable. In light of this, anomalies that have been cited in the literature about play being inconsistent with beliefs (e.g., Costa-Gomes 
and Weizsacker 2006) are not surprising. The evidence is sufficiently convincing at this point, that a reasonable position might be that the use of such procedures yield data that is at best unreliable and at worst misleading. Our own view is more neutral, and one hopes that more reliable methods can be discovered. In the meantime, forecasts elicited directly from players should be interpreted cautiously with the expectation that they may be distorted in some of the ways identified here. 


\section{References}

[1] Aumann, R. J. (1976): "Agreeing to Disagree" Annals of Statistics 4, 1236-1239.

[2] Brier, G. (1950): "Verification of Forecasts Expressed in Terms of Probability" Monthly Weather Review, 78, 1-3.

[3] Costa-Gomes, M. and G. Weizsacker (2006): "Stated Beliefs and Play in Normal Form Games" Working Paper, University of York, U.K.

[4] Croson, R. T. A. (2000): "Thinking like a Game Theorist: Factors Affecting the Frequency of Equilibrium Play" Journal of Economic Behavior and Organization, 41, 299-314.

[5] Dominitz, J. and A. Hung (2004): "Homogeneous Actions and Heterogeneous Beliefs: Experimental Evidence on the Formation of Information Cascades" Working Paper, Carnegie Mellon University, U.S.A.

[6] Dufwenberg, M. and U. Gneezy (2000): "Measuring Beliefs in an Experimental Lost Wallet Game" Games \& Economic Behavior, 30, 163-82.

[7] Geanakoplos, J. D. and H. M. Polemarchakis (1982): "We Can't Disagree Forever" Journal of Economic Theory, 28, 192-200.

[8] Glein, I. N. and J. B. Wallace Jr. (1974): "Probabilistically Answered Examinations: A Field Test" The Accounting Review, 49, 363-366.

[9] Goeree, J., C. Holt, and T. Palfrey (2003). "Risk Averse Behavior in Generalized Matching Pennies Games" Games and Economic Behavior, 45, 97-113.

[10] Good, I. J. (1952): "Rational Decisions" Journal of the Royal Statistical Society B, $14,107-14$. 
[11] Hambleton, R. K., D. M. Roberts, and R. E. Traub (1970): "A Comparison of the Reliability and Validity of Two Methods for Assessing Partial Knowledge on a Multiple-Choice Test" Journal of Educational Measurement, 7, 75-82.

[12] Huck S. and G. Weizsacker (2002): "Do Players Correctly Estimate What Others Do? Evidence of Conservatism in Beliefs" Journal of Economic Behavior and Organization, 47, 71-85.

[13] Kadane, J. and R. Winkler. (1988), "Separating Probability Elicitation from Utilities" Journal of the American Statistical Association, 83, 357-63.

[14] Ledyard J., R. Hanson, and T. Ishikida (2005): "An Experimental Test of Combinatorial Information Markets" Working Paper.

[15] McKelvey, R. D., and T. Page (1986): "Common Knowledge, Consensus, and Aggregate Information" Econometrica, 54, 109-127.

[16] McKelvey, R. D., and T. Page (1990): "Public and Private Information: An Experimental Study of Information Pooling" Econometrica, 58, 1321-1339.

[17] McKelvey, R. D., T. Palfrey, and R. Weber (2000): "The Effects of Payoff Magnitude and Heterogeneity on Behavior in 2x2 Games with Unique Mixed Strategy Equilibria" Journal of Economic Behavior and Organization, 42, 523-48.

[18] Nelson, R. G. and D. A. Bessler (1989): "Subjective Probabilities and Scoring Rules: Experimental Evidence" American Journal of Agricultural Economics, 71, 363-369.

[19] Nielsen, L.T., A. Brandenburger, J. D. Geanakoplos, R. D. McKelvey, and T. Page (1990): "Common Knowledge of an Aggregate of Expectations" Econometrica, 58, 1235-1239. 
[20] Nyarko, Y. and A. Schotter (2002): " An Experimental Study of Belief Learning Using Elicited Beliefs" Econometrica, 70, 971-1005.

[21] Ochs, J. (1995) "Games with Unique Mixed Strategy Equilibria: An Experimental Study" Games and Economic Behavior, 10, 202-217

[22] Offerman, T. and J. Sonnemans (1998): "Learning by Experience and Learning by Imitating Successful Others" Journal of Economic Behavior and Organization, 34, $559-575$.

[23] Offerman, T., J. Sonnemans, and A. Schram (1996): "Value Orientations, Expectations, and Voluntary Contributions in Public Goods" Economic Journal, $106,817-45$.

[24] Palfrey, T. and H. Rosenthal (1991): "Testing Game-Theoretic Models of Free Riding: New Evidence on Probability Bias and Learning" in Laboratory Research in Political Economy (T. Palfrey, ed.), University of Michigan Press:Ann Arbor, $239-67$.

[25] Savage, L. J. (1971): "Elicitation of Personal Probabilities and Expectations" Journal of the American Statistical Association, 66, 783-801.

[26] Seidenfeld, T. (1985): "Calibration, Coherence, and Scoring Rules" Philosophy of Science, 52, 274-294.

[27] Selten, R. (1998): "Axiomatic Characterization of the Quadratic Scoring Rule" Experimental Economics, 1, 43-62

[28] Staël von Holstein, C.-A. S. (1971): "An Experiment in Probabilistic Weather Forecasting" Journal of Applied Meteorology, 10, 635-645. 
[29] Staël von Holstein, C.-A. S. (1972): "Probabilistic Forecasting: An Experiment Related to the Stock Market" Organizational Behavior and Human Performance, 8, 139-158.

[30] Winkler, R. L. (1968): "The Consensus of Subjective Probability Distributions" Management Science, 15, B61-B75.

[31] Winkler, R. L. (1971): "Probabilistic Prediction: Some Experimental Results" Journal of the American Statistical Association, 66, 675-685. 